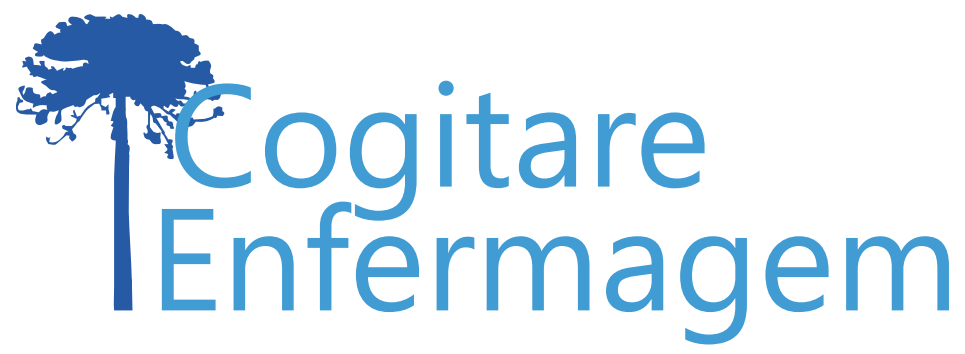

\title{
REIKI NO ALÍVIO DE SINAIS E SINTOMAS BIOPSICOEMOCIONAIS RELACIONADOS À QUIMIOTERAPIA
}

Sieglinder Larissa Beulke ${ }^{1}$, Luciana Vannucci ${ }^{2}$, Léia Fortes Salles ${ }^{3}$, Ruth Natalia Teresa Turrini ${ }^{4}$

\section{RESUMO}

Objetivo: verificar o uso do Reiki no alívio dos sinais e dos sintomas biopsicoemocionais relacionados à quimioterapia.

Metodo: revisão integrativa norteada pela pergunta: A utilização do Reiki pode aliviar alguns sinais e sintomas induzidos pela quimioterapia? A busca foi realizada nas bases de dados Biblioteca Virtual em Saúde (BVS), CINAHL, Cochrane, Embase, PubMed e Scopus sem período de restrição até setembro de 2018. Sete artigos atenderam aos critérios de inclusão.

Resultados: reviram-se quatro ensaios clínicos, dois quase-experimentais e um estudo de caso. Os sinais e sintomas mais estudados foram ansiedade, dor, fadiga, estresse, depressão, humor e bemestar. Pacientes que receberam Reiki relataram melhoria do bem-estar geral, qualidade de vida, dor, depressão, ansiedade, fadiga e humor quando comparados ao grupo controle.

Conclusões: as pesquisas analisadas apresentam evidências que o Reiki pode ser benéfico no alivio dos efeitos colaterais da quimioterapia, o que contribui para mais uma ferramenta para o alívio dos efeitos da quimioterapia.

DESCRITORES: Cuidados de Enfermagem; Revisão; Oncologia; Toque Terapêutico; Terapias Complementares.

COMO REFERENCIAR ESTE ARTIGO:

Beulke SL, Vannucci L, Salles LF, Turrini RNT. Reiki no alívio de sinais e sintomas biopsicoemocionais relacionados à quimioterapia Cogitare enferm. [Internet]. 2019 [acesso em "colocar data de acesso, dia, mês abreviado e ano"]; 24. Disponível em: http://dx.doi.org/10.5380/ce.v24i0.56694.

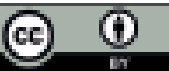

Este obra está licenciado com uma Licença Creative Commons Atribuição 4.0 Internacional. 


\title{
REIKI IN THE RELIEF OF CHEMOTHERAPY-RELATED BIOPSYCHOEMOTIONAL SIGNS AND SYMPTOMS
}

\begin{abstract}
Objective: verify the use of Reiki in the relief of chemotherapy-related biopsychoemotional signs and symptoms.

Method: integrative review guided by the question: Can the use of Reiki relieve some chemotherapy-induced signs and symptoms? The search was undertaken in the databases Virtual Health Library (VHL), CINAHL, Cochrane, Embase, PubMed and Scopus without a restriction period until September 2018. Seven articles complied with the inclusion criteria. Results: four clinical trials, two quasi-experimental studies and one case study were reviewed. The most frequent signs and symptoms mentioned were anxiety, pain, fatigue, stress, depression, mood and well-being. The patients who received Reiki reported improved general well-being, quality of life, pain, depression, anxiety, fatigue and mood in comparison with the control group.

Conclusions: the studies presented evidence that Reiki can exert beneficial effects in the relief of the side effects of chemotherapy, contributing with yet another tool to relieve the effects of the chemotherapy.
\end{abstract}

DESCRIPTORS: Nursing Care; Review; Oncology; Therapeutic Touch; Complementary Therapies.

\section{REIKI EN EL ALIVIO DE SEÑALES Y SÍNTOMAS BIOPSICOEMOCIONALES RELACIONADOS A LA QUIMIOTERAPIA}

\author{
RESUMEN: \\ Objetivo: verificar el uso del Reiki en el alivio de los señales y síntomas biopsicoemocionales \\ relacionados a la quimioterapia. \\ Método: revisión integradora orientada por la pregunta: ¿La utilización del Reiki logra aliviar \\ algunos señales y síntomas inducidos por la quimioterapia? La búsqueda fue ejecutada en \\ las bases de datos Biblioteca Virtual en Salud (BVS), CINAHL, Cochrane, Embase, PubMed y \\ Scopus sin período de restricción hasta septiembre de 2018. Siete artículos cumplieron con \\ los criterios de inclusión. \\ Resultados: fueron revistos cuatro ensayos clínicos, dos casi-experimentales y un estudio de \\ caso. Los señales y síntomas más estudiados fueron ansiedad, dolor, fatiga, estrés, depresión, \\ humor y bienestar. Pacientes que recibieron Reiki relataron mejora del bienestar general, \\ calidad de vida, dolor, depresión, ansiedad, fatigue y humor cuando comparados al grupo de \\ control. \\ Conclusiones: las investigaciones analizadas presentan evidencias de que el Reiki puede ser \\ benéfico en el alivio de los efectos colaterales de la quimioterapia, lo que contribuye hacia \\ una herramienta adicional para aliviar los efectos de la quimioterapia.
}

DESCRIPTORES: Atención de Enfermería; Revisión; Oncología; Tacto Terapéutico; Terapias Complementarias. 
As diversas patologias agrupadas sob o nome de câncer ganham atualmente destaque devido ao crescente envelhecimento da população mundial. Com a melhoria na qualidade de vida obtida pelos avanços da medicina, maior longevidade é alcançada por um número superior de pessoas ao redor do mundo. Consequentemente, mais e mais pessoas atingem idades nas quais o risco de ocorrência de câncer é mais elevado(1).

Doenças causadas por mutações celulares, como é o caso do câncer, necessitam de um tratamento longo, muitas vezes baseado em mais de um método. A quimioterapia (QT) é o tratamento usual de escolha para essa patologia, visto que é um tratamento sistêmico não-específico, baseado na utilização de agentes químicos combinados, visando a destruir as células cancerígenas do portador. Entretanto, como o tratamento não é seletivo, pode afetar também células saudáveis, levando aos efeitos adversos ${ }^{(2-3)}$.

A OT pode ser utilizada junto a tratamentos como a cirurgia e a radioterapia. As técnicas de aplicação, levando em conta esses outros tratamentos, são: curativa, quando se tem apenas a QT como tratamento em busca da cura; adjuvante, quando a QT é o tratamento indicado pós cirurgia; neoadjuvante ou prévia, com a finalidade de reduzir parcialmente o tumor, permitindo a realização da cirurgia e, paliativa, indicada para a melhora da sobrevida do paciente, sem a possibilidade de cura. Dependendo da técnica escolhida, a dosagem recomendada sofre alterações, ocasionando efeitos colaterais diversos ${ }^{(3-4)}$.

Além dos efeitos comuns ao diagnóstico do câncer (estresse, ansiedade, depressão e medo da morte), alguns possíveis efeitos colaterais relacionados à quimioterapia são: dor, fadiga, náuseas e vômitos, constipação ou diarreia, mucosites, ressecamento da pele e queda de cabelos ${ }^{(5-6)}$. Com o intuito de minimizar ou aliviar alguns desses efeitos colaterais, diversos pacientes fazem uso de Práticas Integrativas e Complementares em Saúde (PICS), que aliadas ao tratamento convencional podem propiciar uma melhor qualidade de vida aos pacientes ${ }^{(6-7)}$.

No Brasil, as práticas integrativas e complementares foram integradas ao Sistema Único de Saúde (SUS) no ano de 2006 pela Política Nacional de Práticas Integrativas e Complementares (PNPIC), no entanto somente na Portaria $n^{\circ}$ 849/2017 do Ministério da Saúde, houve a inclusão do Reiki como prática complementar em saúde ${ }^{(8)}$.

As terapias Acupuntura, Aromaterapia, Cromoterapia, Fitoterapia, Florais, Musicoterapia, Reiki e Yoga são as mais comumente utilizadas pelos pacientes em QT como forma de alívio dos efeitos adversos provocados pelo tratamento ${ }^{(6)}$. No presente estudo de revisão integrativa da literatura, optou-se por escolher a utilização do Reiki na redução dos efeitos colaterais da QT.

O Reiki é uma terapia holística baseada no conceito de energia vital (Rei significa universal e Ki, força vital). Idealizada por Mikao Usui no começo do século 20, o Reiki consiste em estimular os canais de energia do paciente por meio da colocação das mãos do praticante em diversas posições sobre ou levemente acima do corpo do paciente. É uma técnica de biocampo utilizada para corrigir os desequilíbrios de energia vital do paciente por não ser invasiva e não necessitar de instalações especiais. Por ser considerada uma intervenção de baixo risco, cresce em popularidade e utilização em diversos hospitais pelo mundo(9).

A literatura cita estudos com aplicação de Reiki em diversas situações, dentre elas, estresse/relaxamento, cura de feridas pela melhora do sistema imune, redução da dor ${ }^{(10)}$, diminuição da ansiedade, insônia e de efeitos colaterais de tratamentos agressivos, entre outros $^{(11)}$. Sua utilização pela enfermagem acrescenta novas possibilidades do cuidar, de maneira não invasiva, simples e segura.

O período de aplicação do Reiki é um momento que favorece uma relação interpessoal de melhor qualidade entre o enfermeiro e o cliente, pois como a linguagem verbal não 
é utilizada, permite outras formas de comunicação ou de percepção que contribuirão de forma relevante para o tratamento e bem-estar deste indivíduo.

Dada a possibilidade de utilização do Reiki, como uma intervenção de enfermagem, esta pesquisa tem por objetivo verificar o uso do Reiki no alívio dos sinais e dos sintomas biopsicoemocionais relacionados à QT por meio de uma revisão integrativa de literatura.

\section{MÉTODO}

Trata-se de uma revisão integrativa de literatura que incluiu as seguintes etapas: identificação do tema e formulação da questão de pesquisa; estabelecimento de critérios para seleção dos estudos e busca na literatura; categorização dos estudos; interpretação dos resultados e síntese do conhecimento ${ }^{(12)}$.

O tema do trabalho relaciona-se com a crescente utilização das PICS por pacientes de câncer e como essa prática é vista nos dias atuais. A questão norteadora escolhida para esse estudo foi: "A utilização do Reiki pode aliviar os sintomas induzidos pela quimioterapia?".

Realizou-se busca nas bases de dados: Biblioteca Virtual em Saúde (BVS), Cumulative Index to Nursingand Allied Health Literature (CINAHL), Cochrane, Embase, PubMed e Scopus, sem período de restrição até setembro de 2018. As palavras-chave utilizadas foram: quimioterapia (chemotherapy no idioma inglês); câncer (cancer) e Reiki (Reiki). A procura considerou a intersecção desses termos na obtenção dos resultados. Foram excluídos das buscas o termo "toque terapêutico" (touch therapy), porque esta técnica energética difere do Reiki. A expressão de busca foi definida da seguinte maneira: (chemotherapy AND cancer AND Reiki) NOT (touch therapy).

\section{RESULTADOS}

Das bases selecionadas, foram obtidos 123 artigos, dos quais 27 eram repetidos. Considerando os respectivos títulos e resumos, outros 71 foram excluídos. Dos 25 restantes, 18 foram excluídos pelos seguintes motivos: comentário sobre artigo que fora excluído, sem conclusão sobre a eficiência do Reiki, abordagem de diferentes afecções além do câncer ou diferentes tratamentos além da QT, uso do Reiki como paliativo sem foco em seus efeitos, e um artigo não foi localizado. A Figura 1 representa o diagrama de fluxo o processo de seleção dos artigos que foram incluídos no estudo. 


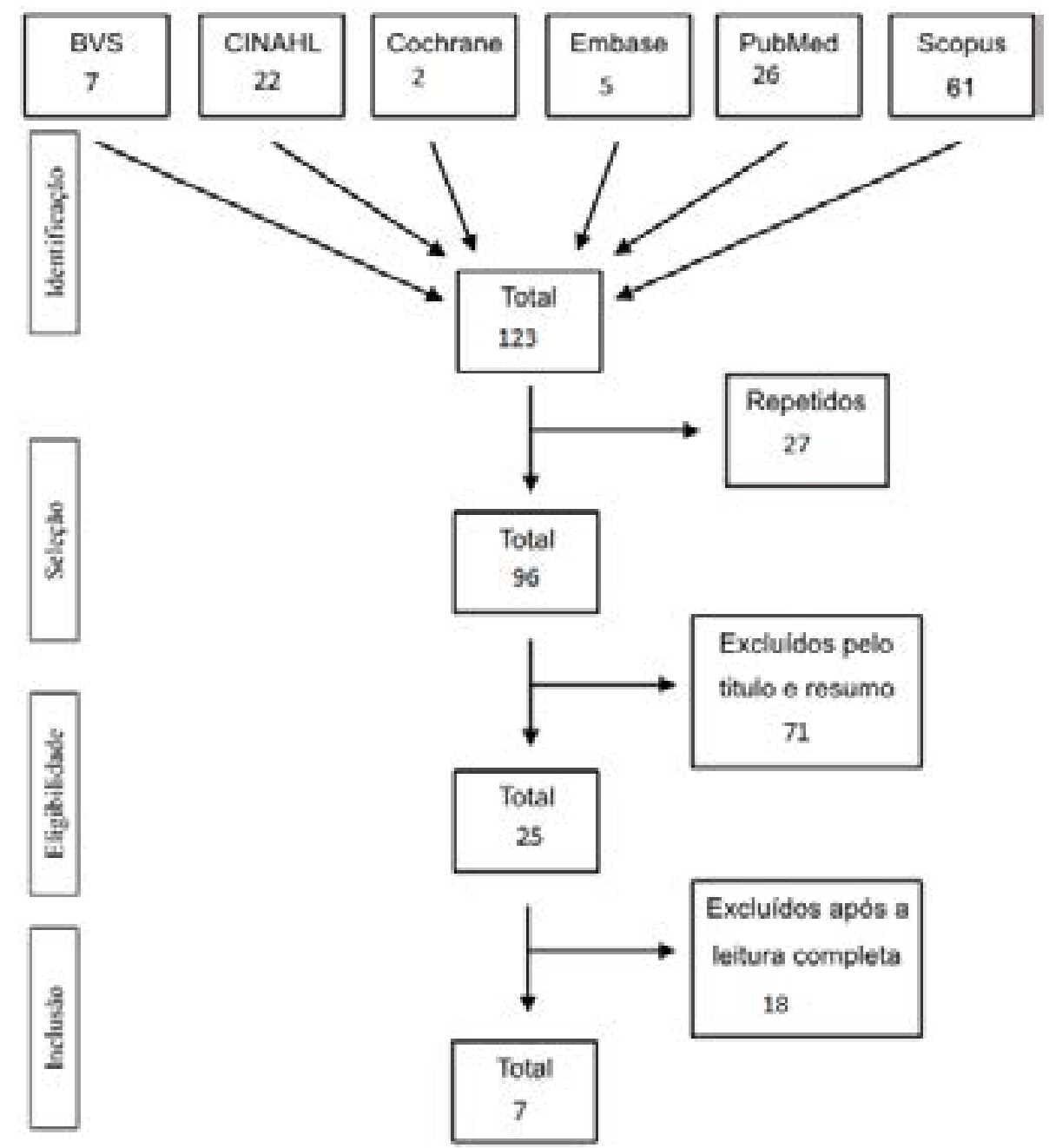

Figura 1 - Diagrama de fluxo do processo de seleção e inclusão dos artigos. São Paulo, SP, Brasil, 2018

Sete artigos foram selecionados para analisar a utilização do Reiki no alívio dos sinais e sintomas biopsicoemocionais relacionados à QT. Desse total, quatro estudos $(57,1 \%)$ foram realizados nos Estados Unidos, um no Canadá (14,3\%), um na Itália (14,3\%) e um na Turquia (14,3\%). Todos os estudos foram realizados nos últimos dez anos, sendo a publicação mais antiga datada de 2007.

Dentre os tipos de pesquisa, seis (86\%) artigos realizaram pesquisas experimentais, sendo quatro estudos ensaios clínicos e outros dois do tipo de intervenção antes e depois. Um artigo (14\%) efetuou uma pesquisa observacional (estudo de caso) sem a presença de grupo de controle (Tabela 1). Dentre os tipos de estudo, todos eram quantitativos e um deles apresentou também uma análise qualitativa.

Tabela 1 - Estudos incluídos segundo autor, tipo de pesquisa, número amostral e principais resultados. São Paulo, SP, Brasil, 2018 (continua)

\section{Autor/ano}

Fleisher KA, Mackenzie

ER, Frankes ES,

Casarett D, Mao JJ. ${ }^{(13)}$

2013
Tipo de Pesquisa

Observacional/

Analítica

(Estudo de caso)

$(n=213)$

\section{Resultados Principais}

Houve declínio de mais de $50 \%$ no estresse, ansiedade, depressão, dor e fadiga. Análises qualitativas reportaram que o Reiki induz ao relaxamento e elevam o bem-estar espiritual. 
Orsak G, Stevens AM, Experimental BrufskyA, Kajumba M, (Ensaio Clínico) Dougall AL. ${ }^{(14)}$ 2015 $(n=36)$
Verificou-se melhora na qualidade de vida e humor nos grupos Acompanhantes e Reiki, quando comparados ao grupo controle.

\section{Catlin A, Taylor-Ford} RL. ${ }^{(15)}$

2011

\section{Experimental (Ensaio Clínico duplo-cego) $(n=189)$}

Os tratamentos com as terapias Reiki e Sham-Reiki aumentaram estatisticamente o conforto e bem-estar dos pacientes pós terapia quando comparados ao grupo controle, embora sem diferença entre a Terapia de Reiki e o Sham-Reiki Placebo.

\begin{tabular}{|c|c|c|}
\hline $\begin{array}{l}\text { Birocco N, Guillame } \\
\text { C, Storto S, Ritorto G, } \\
\text { Catino C, Gir N et al. }{ }^{(16)} \\
2012\end{array}$ & $\begin{array}{l}\text { Experimental } \\
\text { (Estudo antes e } \\
\text { depois) } \\
(n=118)\end{array}$ & $\begin{array}{l}\text { Diminuição da dor e da ansiedade }(p<0,001) \text {, além } \\
\text { de ajudar no bem-estar, relaxamento, alívio da dor, } \\
\text { qualidade do sono e redução da ansiedade. }\end{array}$ \\
\hline
\end{tabular}

\begin{tabular}{ll}
\hline Clark PG, Cortese- & Experimental \\
Jimenez G, Cohen E. ${ }^{(17)}$ & (Antes e depois \\
2012 & com 4 braços \\
& randomizados) \\
& $(\mathrm{n}=9)$ \\
\hline
\end{tabular}

\begin{tabular}{ll}
\hline $\begin{array}{l}\text { Tsang KL, Carlson LE, } \\
\text { Olson K. }{ }^{(18)}\end{array}$ & $\begin{array}{l}\text { Experimental } \\
\text { (Ensaio Clínico } \\
2007\end{array}$ \\
$\begin{array}{l}\text { Duplo-cego) } \\
(n=16)\end{array}$ \\
\hline $\begin{array}{l}\text { Demir M, Can G, } \\
\text { Kelam A, Aydiner A. }{ }^{(19)} \\
2015\end{array}$ & $\begin{array}{l}\text { Experimental } \\
(\text { Ensaio Clínico) } \\
(n=18)\end{array}$
\end{tabular}

Nenhuma terapia (Yoga, Reiki, Meditação) mostrou diferença estatística significativa após a terapia. No entanto, observou-se algum efeito positivo do uso do Reiki, trazendo benefícios físicos e psicológicos aos participantes que receberam tal terapia.

Houve maior redução da fadiga, dor e ansiedade, estatisticamente significativa no grupo experimental em relação ao grupo controle.

Ao final do experimento os níveis de dor, fadiga e estresse foram menores nos indivíduos do grupo experimental e maiores no grupo controle.

Destaca-se a variedade de sintomas estudados, bem como os diferentes tipos de câncer, faixas etárias, tamanho de amostra, quantidade e duração das sessões de Reiki dos estudos selecionados.

Os sintomas provenientes da QT mais investigados foram: fadiga, dor, estresse, ansiedade, confusão, depressão, bem-estar, humor e neuropatia periférica, sendo os três primeiros os mais pesquisados. A fadiga apareceu em cinco $(71 \%)$ dos sete artigos examinados e a ansiedade e a dor em quatro artigos (57\%).

Entre os diversos tipos de câncer considerados, o principal foi o câncer de mama ( $n=4$; $57 \%)$. Outros tipos foram o câncer colorretal, pulmonar, leucemia e geniturinário(14,16-19). Vale a ressalva que dois estudos não especificaram o tipo de câncer ${ }^{(13,15)}$.

O tamanho das amostras foi variável, de 16 a 213 participantes, tendo estudos com amostras superiores a $180,{ }^{(13,15)} 118^{(16)}$ e estudos com um reduzido número de participantes, inferiores a $40^{(14,17-19)}$. A faixa etária predominante supera os 60 anos de idade, sendo a idade média geral dos participantes dos estudos igual a 65 anos (a menor idade relatada nos estudos foi de 33 anos e a maior foi de 84 anos).

Uma síntese das informações sobre as técnicas de Reiki utilizadas nos estudos bem como o nível de conhecimento dos praticantes que aplicaram as técnicas e o tempo médio das sessões da terapia oferecida aos participantes dos estudos é apresentada na Tabela 2. 
Tabela 2 - Informações adicionais sobre a intervenção com Reiki nas pesquisas selecionadas. São Paulo, SP, Brasil, 2018

\begin{tabular}{|c|c|c|c|c|}
\hline Autores & $\begin{array}{l}\text { Nível do } \\
\text { Praticante }\end{array}$ & $\begin{array}{l}\text { Experiência do } \\
\text { Praticante }\end{array}$ & $\begin{array}{c}\mathbf{N}^{\circ} \\
\text { sessões }\end{array}$ & $\begin{array}{l}\text { Tempo médio } \\
\text { da sessão }\end{array}$ \\
\hline $\begin{array}{l}\text { Fleisher KA, Mackenzie ER, Frankes ES, } \\
\text { Casarett D, Mao JJ.(13) }\end{array}$ & $\begin{array}{l}\text { Mestre-Praticante } \\
\text { Reiki - Nível } 2\end{array}$ & $\begin{array}{l}\text { avaliação das } \\
\text { capacidades e } \\
\text { treinamento }\end{array}$ & 1 a 11 & 10 a $30 \mathrm{~min}$ \\
\hline $\begin{array}{l}\text { Orsak G, Stevens AM, Brufsky A, } \\
\text { Kajumba M, Dougall AL. }{ }^{(14)}\end{array}$ & $\begin{array}{l}\text { Mestre-Praticante } \\
\text { Reiki - Nível } 2\end{array}$ & - & - & $30 \mathrm{~min}$ \\
\hline Catlin A, Taylor-Ford RL. ${ }^{(15)}$ & Mestre de Reiki & $\begin{array}{l}\text { experiente e } \\
\text { treinado }\end{array}$ & - & $20 \mathrm{~min}$ \\
\hline $\begin{array}{l}\text { Birocco N, Guillame C, Storto S, Ritorto } \\
\text { G, Catino C, Gir N et al. }{ }^{(16)}\end{array}$ & - & 2 anos & 1 a 4 & $30 \mathrm{~min}$ \\
\hline $\begin{array}{l}\text { Clark PG, Cortese-Jimenez G, Cohen } \\
\text { E.(17) }\end{array}$ & Mestre de Reiki & - & 6 & $60 \mathrm{~min}$ \\
\hline Tsang KL, Carlson LE, Olson K..$^{(18)}$ & Mestre em Reiki & 10 anos & 7 & $45 \mathrm{~min}$ \\
\hline Demir M, Can G, Kelam A, Aydiner A. ${ }^{(19)}$ & $\begin{array}{l}\text { Praticantes } \\
\text { Reiki - Nível } 2\end{array}$ & 4 anos & 5 & $30 \min$ \\
\hline
\end{tabular}

O número de sessões de Reiki foram de 1 a 11 e o tempo de duração das sessões de 10 a 60 minutos, mas dois estudos não mencionaram a quantidade de sessões de Reiki ${ }^{(14-15)}$. Os estudos também não foram uniformes no detalhamento descritivo dos praticantes do Reiki.

\section{DISCUSSÃO}

Todas as pesquisas experimentais utilizaram modelos de questionários já existentes para avaliar o uso do Reiki no alívio dos sinais e dos sintomas biopsicoemocionais relacionados à QT. Além disso, 62,5\% dos estudos possuíam um grupo controle para posterior comparação dos resultados da aplicação de Reiki aos pacientes. Embora utilizassem diferentes variáveis, instrumentos de pesquisa e modo de avaliação dos dados, todos avaliaram os efeitos do Reiki considerando as respostas dos participantes no período da QT, antes e depois das sessões. Avaliações sociodemográficas foram realizadas para verificar se havia alguma diferença nos grupos considerados nesses estudos, nenhuma diferença estatística foi observada ${ }^{(14-19)}$.

O estudo(15) que realizou a intervenção com Reiki e Sham-Reiki (placebo: enfermeira ao lado do paciente) para compará-los ao grupo controle incluiu um bom número de participantes ( $n=63$ por grupo) e utilizou o Questionário do Conforto da Cura pelo Toque (Healing Touch Comfort Questionnaire, HTCQ) e a Escala Análoga do Bem-Estar (WellBeing Analog Scale, WBAS) para avaliar o desfecho da intervenção. Como efeito geral, o Sham-Reiki e o Reiki apresentaram melhora no bem-estar e no conforto geral dos pacientes se comparados ao grupo de controle. Entretanto não apresentaram diferença perceptível entre eles. Como resultado geral, os autores concluíram que a presença de uma enfermeira, mesmo sem tocar o paciente, traz um benefício no conforto e bem-estar geral dos pacientes.

Três estudos ${ }^{(14,17-18)}$ utilizaram versões da escala Functional Assessment of Cancer Therapy, que inclui aspectos funcionais e variáveis de qualidade de vida para avaliar os efeitos do Reiki. Um deles ${ }^{(14)}$ utilizou o Functional Assessment of Cancer Therapy: Breast Cancer Version, FACT-B, uma versão para as pessoas com câncer de mama, além da Escala 
de Angústia de Sintomas (Symptom Distress Scale, SDS), a escala de Mudanças de Humor versão curta (Profile of Mood States - Short Form Questionnaire, PMS-SF) e perguntas para os que receberam a intervenção com Reiki como "A intervenção foi relaxante?" e "Algum problema resultante da terapia com Reiki?" Diferenças significativas foram observadas na escala de qualidade de vida e na escala de humor geral e para o domínio confusão da PMS-SF, quando considerada a interação com o tempo (número de sessões de QT), com melhores resultados para o grupo Reiki e o grupo Acompanhantes comparados ao controle. O grupo Acompanhantes apresentou melhores resultados que o grupo Reiki.

Autores $^{(17)}$ utilizaram grupo controle e de Reiki com sete participantes e analisaram variáveis como: angústia pelo Brief Symptom Inventory (BSI); qualidade de vida ou neurotoxicidade pela Avaliação Funcional das Terapias do Câncer (Functional Assessment of Cancer Therapies - Gynecologic Oncology Group-Neurotoxicity Scale, FACT/GOG-Ntx) e uma escala de atenção plena (Mindful Attention Awareness Scale, MAAS). Os resultados encontrados deram indícios positivos da utilização do Reiki no tratamento das neuropatias periféricas induzidas pela QT. O uso do Reiki trouxe benefícios físicos e psicológicos aos participantes que se submeteram a tal terapia.

A investigação(18) do efeito do Reiki sobre a fadiga, dor e ansiedade em indivíduos submetidos à OT utilizou instrumentos de medida da qualidade de vida: Avaliação Funcional das Terapias do Câncer para fadiga e geral (Functional Assessment of Cancer Therapy: Fatigue - FACT-F/Functional Assessment of Cancer Therapies- General - FACT-G) e escalas analógicas para dor, cansaço e ansiedade. O grupo experimental foi submetido a sete sessões de Reiki e o grupo controle ao mesmo número de sessões de descanso. Todos os participantes responderam os instrumentos de avaliação antes e após cada intervenção. Houve diferença significativa entre os resultados dos dois grupos com maiores reduções da fadiga, dor e ansiedade e melhora da qualidade de vida no grupo experimental em relação ao controle.

Uma Escala Análoga Visual foi empregada para avaliar a dor e ansiedade ao final das sessões de uma das pesquisas ${ }^{(16)}$. Os participantes descreveram sintomas físicos sentidos durante a sessão (calor ou frio, relaxamento ou tensão, bem-estar ou desconforto) e sua localização no corpo. Todos se submeteram a pelo menos uma sessão de Reiki (variação de dois a quatro). Os efeitos observados foram: diminuição da dor e da ansiedade, e relatos como: ajuda no bem-estar, relaxamento, alívio da dor, qualidade do sono e redução da ansiedade. As sessões foram consideradas úteis para melhorar o relaxamento, bem-estar e ansiedade, com proporções de $70 \%$ ou mais, e inferiores a $50 \%$ em condições físicas como dor e sono.

No estudo randomizado e controlado(19) foi avaliado o efeito do Reiki a distância durante 30 minutos sobre a fadiga, dor e estresse de pacientes internados em unidade oncológica e recebendo quimioterapia. O grupo controle recebeu apenas os cuidados usuais. Ao final da intervenção, os níveis de dor, estresse e fadiga aumentaram no grupo controle e diminuíram no grupo experimental, com resultados significativos.

No estudo de caso(13) com uma ou mais sessões de Reiki, utilizou-se uma versão modificada do Distress Thermometer (DT), com a pergunta do tipo Likert "quão angustiado se sentiu durante a última semana incluindo o dia de hoje?" A escala DT também foi expandida para incluir a avaliação de depressão, ansiedade, dor e fadiga, além da angústia. Os autores observaram um declínio de pelo menos $50 \%$ na angústia, ansiedade, depressão, dor e fadiga, e a análise de conteúdo da pergunta aberta evidenciou que o Reiki induz ao relaxamento e elevam o bem-estar espiritual. Vale a ressalva que não houve gravação da resposta da pergunta aberta e que a escala DT não foi completamente validada para estudos de mudanças temporárias na angústia seguida da intervenção.

As pesquisas analisadas ${ }^{(13-19)}$ apresentaram evidências que o Reiki pode ser benéfico no tratamento dos sinais e sintomas biopsicoemocionais oriundos da quimioterapia por ser um método não invasivo, de fácil aplicação e não necessitar de instalações especiais. Entretanto, métodos mais rigorosos e reprodutíveis, com ensaios randomizados e melhor 
descrição das intervenções e dos provedores das terapias alternativas e complementares, merecem ser conduzidos.

Os estudos incluídos apresentaram algumas limitações como: a heterogeneidade dos artigos analisados em relação à metodologia, tipo de câncer e sinais e sintomas investigados, tamanho da amostra, idade dos participantes e número e tempo de duração das sessões de Reiki. O efeito placebo e a presença de uma pessoa para confortar o participante devem ser melhor investigados e, também, não se encontraram na literatura estudos que mostrassem o efeito do Reiki em adolescentes e crianças.

\section{CONCLUSÃO}

As pesquisas analisadas apresentam evidências que o Reiki pode ser benéfico no alívio dos efeitos colaterais da quimioterapia. Os pacientes que receberam o tratamento com o Reiki relataram melhora no bem-estar geral e de alguns sintomas, mas nem todos os ensaios clínicos apresentaram resultados significativos. A melhora de sinais e sintomas como dor, fadiga, estresse, ansiedade sugerem que esta prática pode ser introduzida na prática assistencial ou recomendada a pacientes submetidos a quimioterapia ambulatorial.

\section{REFERÊNCIAS}

1.Bennett MI, Flemming K, Closs SJ. Education in cancer pain management. CurrOpinSupportPalliatCare. [Internet]. 2011 [acesso em jul 2016]; 5(1). Disponível em: http://dx.doi.org/10.1097/SPC.0b013e328342c607.

2.Smith PJ,ClavarinoA, Long J, Steadman KJ. Why do some cancer patients receiving chemotherapy choose to take complementary and alternative medicines and what are the risks? AsiaPac J ClinOncol[Internet]. 2014 [acesso em jul 2015];10(1). Disponível em: http://dx.doi.org/10.1111/ajco.12115.

3.Schwab M. Encyclopedia of Cancer. 3. ed. Berlim: Springer; 2011.

4.Silva JMC, Sousa PP. Estrategias para el autocuidado de las personas con cáncer que reciben quimioterapia/ radioterapia y su relación con el bienestar. Enferm. glob[Internet]. 2015[acesso em jan2017];14(1). Disponível em: http://revistas.um.es/eglobal/article/view/eglobal.14.1.206591/169981.

5.Singh P, ChatuverdiA. Complementary and Alternative Medicine in Cancer Pain Management: A Systematic Review. Indian J PalliatCare. [Internet]. 2015[acesso emago2016];21(1). Disponível em: http:// dx.doi.org/10.4103/0973-1075.150202.

6.Jaconodino $\mathrm{CB}$,Amestoy SC,Thofehrn MB. A utilização de terapias alternativas por pacientes em tratamento quimioterápico. Cogitare enferm. [Internet]. 2008 [acesso em jun 2016];13(1). Disponível em: http://dx.doi.org/10.5380/ce.v13i1.11953.

7. Costa AIS, Reis PED. Técnicas complementares para controle de sintomas oncológicos. Rev Dor.[Internet]. 2014 [acesso em maio 2016];15(1). Disponível em: http://dx.doi.org/10.5935/1806-0013.20140014.

8. Sousa IMC, Bodstein RCA, Tesser CD, Santos FAS, Hortale VA. Integrative and complementary health practices: the supply and production of care in the Unified National Health System and in selected municipalities in Brazil. Cad Saude Publica [Internet]. 2012 [acesso em jun 2016];28(11). Disponível em: http://dx.doi.org/10.1590/S0102-311X2012001100014.

9. Quest P, Roberts K. The Reiki- Manual:a training guide for Reiki students, practitioners, and masters. London: Hachette Digital: 2011.

10. Rindfleisch JA. Biofield therapies: energy medicine and primary care. Prim Care. [Internet].2010 [acesso em jul 2016];37(1). Disponível: http://dx.doi.org/10.1016/j.pop.2009.09.012.

11. Vannucci L, Salles LF, Minami A. Reiki e Enfermagem. In: Salles LF, Silva MJP. Enfermagem e as práticas 
complementares em saúde. São Paulo: Yendis; 2011.

12. Mendes KDS, Silveira RCCP, Galvão CM. Revisão integrativa: método de pesquisa para a incorporação de evidências na saúde e na enfermagem. Texto contexto- enferm. 2008;17(4): 758-64.

13. Fleisher KA, Mackenzie ER,Frankes ES,Casarett D, Mao JJ. Integrative Reiki for cancer patients: a program evaluation. IntegrCancerTher[Internet]. 2014 [acesso em jul 2016];13(1). Disponível em: http:// dx.doi.org/10.1177/1534735413503547.

14. OrsakG, Stevens AM, BrufskyA, Kajumba M, Dougall AL. The effects of Reiki therapy and companionship on quality of life, mood, and symptom distress during chemotherapy. Evid Based Complement Alternat Med. [Internet]. 2015 [acesso em jan 2017];20(1). Disponível em: http://journals.sagepub.com/doi/ $\mathrm{pdf} / 10.1177 / 2156587214556313$.

15. Catlin A,Taylor-Ford RL. Investigation of standart care versus sham Reiki placebo versus actual Reiki therapy to enhance comfort and well-being in a Chemoterapy Infusion Center. Oncol Nurs Forum[Internet]. 2011 [acesso em jul 2016];38(3). Disponível em: http://dx.doi.org/10.1188/11.ONF.E212-E220.

16. Birocco N, Guillame C, Storto S, Ritorto G, Catino C, Gir Net al..Theeffects of Reiki therapy on pain and anxiety in patients attending a day oncology and infusion services unit. J PalliatMed[Internet].2012 [acesso em jul 2016];29(4). Disponível em: http://dx.doi.org/10.1177/1049909111420859.

17. Clark PG, Cortese-Jimenez G, Cohen E. Effects of Reiki, Yoga, or meditation on the physical and psychological symptoms of chemotherapy-induced peripheral neuropathy - a randomized pilot study. J Evid Based Integr Med [Internet].2012 [acesso em maio 2016];17(3). Disponível em: http://journals.sagepub. com/doi/abs/10.1177/2156587212450175.

18. Tsang KL, Carlson LE, Olson K. Pilot crossover trial of Reiki versus rest for treating cancer-related fatigue. IntegrCancerTher [Internet]. 2007 [acesso em jan 2017]; 6(1). Disponível em: https://doi. org/10.1177/1534735406298986.

19. Demir M, CanG, Kelam A, Aydiner A. Effects of distant Reikionpain, anxiety and fatigue in oncology patients in Turkey: apilotstudy. Asian PacifJ Cancer Prev.[Internet]2015;16(12)[acessoemjan 2017]. Disponível em: http://journal.waocp.org/article 31175 4410b9aee922916320996fa472723164.pdf.

Recebido: 04/12/2017

Finalizado: 17/12/2018

Autor Correspondente:

Léia Fortes Salles

Universidade de São Paulo

R. Faustolo, 656 - 05041-000 - São Paulo, SP, Brasil.

E-mail: leia.salles@usp.br

\section{Contribuição dos autores:}

Contribuições substanciais para a concepção ou desenho do estudo; ou a aquisição, análise ou interpretação de dados do estudo - SLB, LV, LFS, RNTT

Elaboração e revisão crítica do conteúdo intelectual do estudo - LV, LFS

Aprovação da versão final do estudo a ser publicado - LV, LFS, RNTT 